\title{
Squamous Cell Carcinoma Developing in Mature Cystic Teratoma of the Ovary: A Rare Case
}

\author{
Thaker Shilpa
}

Received: 10 September 2009/Accepted: 24 May 2012/Published online: 22 March 2013

(C) Federation of Obstetric \& Gynecological Societies of India 2013

\section{Introduction}

Squamous cell carcinoma (SCC) is the most common type, that is 80-90\%, of malignant tumor developing in benign cystic teratoma. Others are adenocarcinoma, undifferentiated sarcoma and carcinoma, and papillary carcinoma.

Squamous cell carcinoma arising from mature cystic teratoma is a rare pathologic event accounting for only $1-2 \%$ of cases.

\section{Case Report}

A postmenopausal 50-year-old woman presented with the complaint of on and off pain in the abdomen for the past 2-3 months, nausea, vomiting, and loss of appetite for the past 1 month, and loss of weight for the past 3 months. She did not have any other complaints. She was menopausal for the past 5 years. On clinical examination, she had a bloated abdomen with a huge mass in it extending up to the epigastric region. All other clinical vital signs were normal. Her hemoglobin was $8.8 \mathrm{gm} \%$ and ESR $82 \mathrm{~mm} / \mathrm{h}$. Other blood and urine investigations were within normal limits.

Thaker S. ( $₫)$, Consultant OBS. \& GYN and Infertility Specialist

The Rathod Hospital, Chaudhary High School Circle, Rajkot 360 001, Gujarat, India

e-mail: dr.shilpathaker@gmail.com
Ultrasound examination showed a large mixed echogenic ovarian cyst from the pelvis to the upper abdomen, which was mainly cystic with a small solid part.

Exploratory laparotomy was performed which revealed a cystic ovary measuring approximately $30 \mathrm{~cm}$ in diameter on the right side. The cyst was adherent to the intestine. The left ovary was normal and an oophorectomy was done. There were coconut-sized retroperitoneal glands adherent to the inferior vena cava and aorta. That mass was inoperable. FNAC from the glands was done. There were no ascites or secondaries in the liver and other glands and no intestinal obstruction (Figs. 1, 2).

Gross inspection showed a pink, shiny, and wellcircumscribed right ovarian cyst totally measuring $29 \times 15 \times 11 \mathrm{~cm}$ and weighing about $5.20 \mathrm{~kg}$. On cutting, the mass showed a dirty and pultaceous material admixed with hairs. The inner surface was predominantly cystic with some solid areas having a yellow-white, firm, and granular cut surface. Microscopic examination showed stratified and squamous epithelial lining covering the cyst. Underneath, heterogeneous tissue components were identified in the stroma consisting of epidermal appendages, intestinal glands, mature neural tissue, hair follicles, and cartilaginous fragments. Histopathologic examination of the solid areas showed features of SCC.

The postoperative period was uneventful and she was discharged on the 5th day. She was advised chemotherapy and irradiation. However, the patient was not ready for any adjuvant treatment and she did not return for follow-up. 


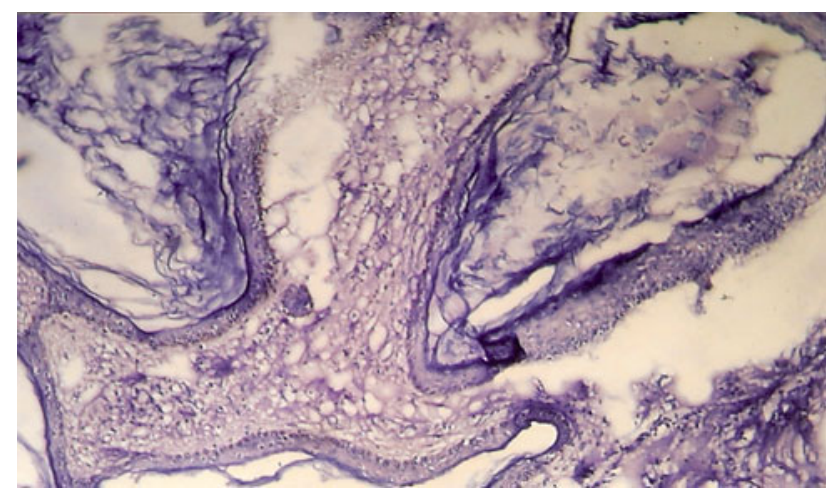

Fig. 1 Histopathology slide

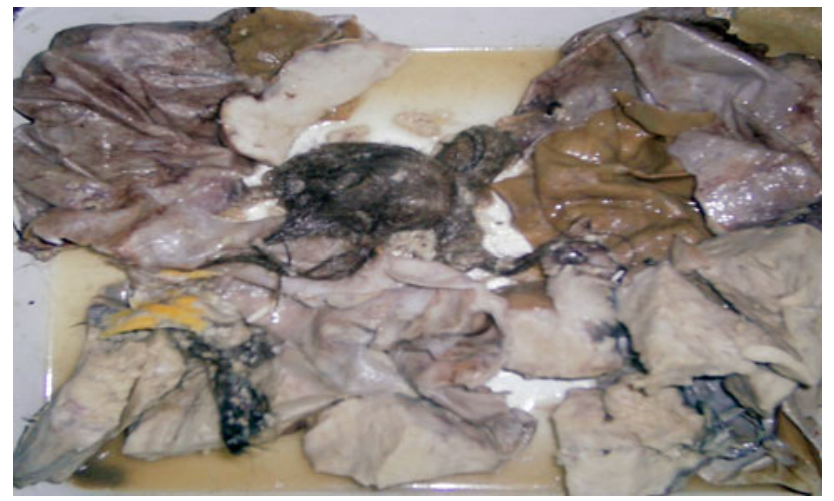

Fig. 2 Gross appearance of tumor

\section{Discussion}

Mature cystic teratoma of the ovary develops from three germ layers (endoderm, mesoderm, and ectoderm). It accounts for $10-20 \%$ of all ovarian tumors in woman of reproductive age [1], while malignant transformation of mature teratoma is more common after the age of 50. The incidence of malignant transformation of mature cystic teratoma of the ovary is rare, i.e., $1-2 \%[1,2]$, and undiagnosed preoperatively most of the time. In such kind of cases, there are no signs and symptoms suggesting malignancy. The common symptoms are abdominal pain, abdominal or pelvis mass, abdominal distention, or bloated abdomen as those are caused by benign cysts [3]
Malignancy in mature teratoma is difficult to diagnose clinically, but there are some risk factors like age, size of tumor, and serum tumor markers for malignancy in mature cystic teratoma. SCC in mature cystic teratoma has been observed usually after menopause, although it has been reported sometimes in young patients around 30 years of age $[4,5]$. The age of the patient in our case was 50 years, i.e., in the usual range of this disease.

Tumor size is another criterion to predict malignancy. In our case, the tumor diameter was around $30 \mathrm{~cm}$, which is larger than a typical benign cyst. In general, it is recommended that a diameter equal to or greater than $10 \mathrm{~cm}$ or a tumor demonstrating rapid growth should prompt suspicion.

Prognosis of these tumors is very poor with the five-year survival rate only 15-30\% [1]. Better prognosis has been reported in the case of SCC compared with adenocarcinoma or sarcoma [1].

The main treatment for mature cystic teratoma with malignant transformation is surgery, and total removal of the genital organs should be the choice for postmenopausal woman. Postoperative single-agent or combination chemotherapy, radiotherapy, or a combination of the both were evidenced, but the results were variable, and so optimal adjuvant therapy for squamous cystic teratoma has not yet been established [4].

In conclusion, when older patients present with a dermoid cyst larger than the usual cyst, this rare type of tumor should be kept in mind.

\section{References}

1. Kurman R, Blaustein's J. Pathology of female genital tract. 5th ed. New York: Springer; 2002.

2. Curling ON, Potsides PN, Hudson CN. Malignant change in benign cystic teratoma of the ovary. $\mathrm{Br} \mathrm{J}$ Obstet Gynecol. 1979;86(5):399-402.

3. Spannuth WA, Rocconi RP, Kirfy TO, et al. Gamma mode of infiltration associated with poor prognosis in malignant teratoma of the ovary: a case report: gynecology. Oncology. 2005;98:155-7.

4. Kurte JE, Jaeck D, MAliosel F, et al. Combined modality treatment for malignant transformation of a benign ovarian teratoma. Gynecol Oncol. 1999;73:319-21.

5. Wen KC, Chen P. Poor prognosis of intraoperative rupture of mature cystic teratoma with malignant transformation. Taiwan $\mathrm{J}$ Obstet Gynecol. 2006;45:253-5. 